\title{
NUMERICAL STUDY OF THE SEPARATION EFFICIENCY AND HEAT EXCHANGE PERFORMANCE IN A COMPLEX GAS-SOLID SEPARATOR
}

\author{
FRANCESCO MARIANI, FRANCESCO RISI \& CLAUDIO POGGIANI \\ Department of Engineering, University of Perugia, Italy.
}

\begin{abstract}
This work presents a study on a gas-solid cyclone separator used in a complex cement production plant. The main objective of the study is based on the performance evaluation and optimization of the cyclone separator in terms of particle separation and heat transfer efficiencies, while keeping pressure losses under control. The thermal interaction is between two gas-solid mixtures, one at $850^{\circ} \mathbf{C}$ and the other at $600^{\circ} \mathbf{C}$, respectively. The solid phase consists mostly of calcium carbonate subsequently intended to the so-called baking process for the production of clinker and ultimately cement. A first model has been setup using experimental data as boundary conditions to assess the physical model behavior and the CFD solver parameters. After that, five additional models with different geometries have been analyzed to evaluate the influence of the vortex finder $(v f)$ length on the separation efficiency and on the heat exchange performance. By increasing the length of the $v f$, the results show a global improvement in the separation efficiency of up to 5\% if compared to the geometry without the $v f$. Further, the increase in the $v f$ length determines a monotonic decrease of temperature at the exit but a monotonic increase of pressure losses. In the second part of this work, using one of the previous models with $v f$, a study of the influence of the particle diameter on the separation efficiency has been performed. The increase of particle diameter causes an increase of the separation and thermal exchange performance, decreasing at the same time the pressure drop. The numerical approach for all the cases is based on implicit unsteady simulations using the Eulerian Multiphase model.
\end{abstract}

Keywords: computational fluid dynamics, cyclone, Eulerian multiphase, gas-solid heat exchange, geometrical optimization.

\section{INTRODUCTION}

\subsection{The industrial process}

The present study is a step of a longer activity aimed at the study of possible technological solutions that allow to optimize the heat exchange between a flow of gas at high temperature (about $900^{\circ} \mathrm{C}$ ) and the raw material powder (consisting largely of calcium carbonate), to be submitted to the cooking process for the production of "clinker", and then of cement. Much of this heat exchange takes place in a preheating tower constituted by cyclones and connection pipes: the flow of hot air from the oven meets, in countercurrent, the raw material to be preheated. Current technologies provide the heat exchange between gas and raw materials takes place in a very limited area of the circuit which constitutes the preheating tower, which is in the connecting lines between the various cyclones. In these areas mixing takes place (in equi-current) between the gas and the powder of raw material, which allows a certain heat 
transfer. In the cyclones, which constitute the main part of the structure, any important heat exchange does not take place because the gas-powdery material separation is almost immediate; on the other hand, the separation process is also very important for a good thermal efficiency of the system. The introduction of the suspension preheater, in the early thirties, was an important evolution of the clinkerization process. The dried raw meal is preheated and even partially calcined (dry/semi-wet processes) while is held in suspension with the hot gases coming from the rotary kiln. At least in theory, a wider contact surface allows a heat exchange nearly complete. There are several preheating systems in suspension: Fig. 1a. These systems usually have four to six stages of cyclones, which are arranged one over the other to form a tower with a height varying from 50 to $120 \mathrm{~m}$. The stadium on top can be constituted by two parallel cyclones for a better separation of the powder. The gas exhausted from the furnace flows through the various stages of cyclones from the bottom upwards. The mixture of finely ground raw meal and dry mixes with the gas before the highest stage of cyclones; it is separated from the gas in the cyclone and rejoins it before the next stage of cyclones. This procedure is repeated for each stage until the material is discharged from the last cyclone (the lowest), into the rotary kiln. This cyclical process of mixing, separation and re-mixing occurs at high temperature to optimize the heat transfer. The cement is produced by a process particularly energy-intensive: in fact, each tonne of finished product requires about 120 electric $\mathrm{kWh}$ and $6 \times 10^{5}$ thermal kcal. A standard plant, which produces about $10^{6} \mathrm{t} / \mathrm{year}$, employs about $12 \times 10^{4}$ electric MWh/y and $6 \times 10^{5}$ thermal Gcal/y (equivalent to $8 \times 10^{4}$ ton of $\mathrm{coal} / \mathrm{y}$ ). The thermal energy is used for the cooking of the semi-finished product (clinker) and is mainly obtained by burning petroleum coke (about $8,200 \mathrm{kcal} / \mathrm{kg}$ ). For a standard production of about 125 tons/hour of clinker are burned about 13 tons/hour of petroleum coke; therefore, it is very important to optimize the heat consumption of the firing. The insertion, upstream of a moving grate oven (where takes place the final part of the cooking process), of a system of cyclones (preheating tower), reduces the heat consumption by more than 1,200 $\mathrm{kcal} / \mathrm{kg}$ clinker to about $850 \mathrm{kcal} / \mathrm{kg}$. This change allows you to recover some of the heat from the combustion gases (about $2 \times 10^{5} \mathrm{nmc} / \mathrm{h}$ at $900^{\circ} \mathrm{C}$ ). Despite this, the gases leave the circuit at a temperature of about $330^{\circ} \mathrm{C}-380^{\circ} \mathrm{C}$.

\subsection{State of the art}

In general, the task of a cyclone is the removal of solid particles from a gas with a consequent beneficial effect on the air pollution control. At this aim, a lot of studies available in literature [1-15], developed by many authors, have been widely studied, both numerically and experimentally, with the main goal of increasing the separation efficiency. Multiple studies [1-8] demonstrated the decisive influence of both operating parameters and design on the separation efficiency and pressure drop.

The analyzed models are generally constituted by only one cyclone, with a traditional circular cross section and a tangential inlet. In [1] the authors studied the influence of cylinder shape on separation efficiency; they highlight the influence of the size and shape of the cylinder on the core-annulus shape inside the cyclone. This shape plays a very important role on the global flow and particle separation. In [3], with both experimental and numerical approaches, the authors analyzed the effect of a prolonged cyclone, obtained attaching vertical tubes of different length at the bottom of the particle outlet, on the separation performance. In, Lim et al. [2] have highlighted how the vortex finder plays a critical role in defining the flow field inside the cyclone; at this aim, they took measures to check the particle separation 
performance employing vortex finders of different lengths. In [4], the authors show the influence of the bottom-contracted and edge-sloped vent-pipe on the separation efficiency and pressure drop of a cyclone separator under different vent-pipe insert depth and different orientation of the sloped edge. In the papers $[5,6]$, by experimental tests, the authors have shown that it is possible to decrease pressure drop and improve the separation efficiency using double inlets. In Zheng et al. [7], presented an experimental work that evaluates, inter alia, the variation of separation efficiency and pressure drop, with different combinations of inlet forms and inlet air speed. Bernardo et al. [8] analyzed the effects of the inlet section angles on the fluid dynamics inside the cyclone and on the performance parameters: collection efficiency and pressure drop. The numerical results show that the value for overall collection efficiency increased to $77.2 \%$ for the 45 -inlet section angle, while that for the normal inlet duct was $54.4 \%$ under the same operating conditions. The performance of a water-cooled square cyclone was compared in [16] with that of other cyclones through fly ash analysis. The results showed that the overall performance of the square cyclone in such capacity is compatible to that of the round cyclone (pressure drop less than $800 \mathrm{~Pa}$ ). Yaxin et al. [17] numerically investigated a square cyclone separators with three types of inlet configuration. The resulting velocity, separation efficiency and pressure drop were verified by comparison with experimental data. Results showed that inlet configurations influenced the turbulent dynamics in the cyclone and led to different pressure drop and separation efficiency. Inter alia, the authors proved the separator with single normal inlet (SNI), had the best separation efficiency and the maximum pressure drop. Furthermore, when a baffle was installed in the inlet of separator SNI, the pressure drop increased by about $191 \%$.

For further improvement of cyclone performance it is mandatory to understand turbulent dynamics and the separation process. The CFD-3D analysis is an efficient and economical approach of understanding the fluid dynamics process in complex plants, providing a precious way to evaluate how it is affected by changes of operating conditions and geometric shapes. At this aim, the $C F D$ approach has been used by many authors $[1,8-15]$; the papers deal with issues relative to the flow of gas-solid suspensions predicting solid separation efficiency, at different operating conditions, in good agreement with the experimental evidences. Furthermore, in [13-15] the authors proved as the Reynolds Stress Model is the suitable turbulent model for cyclone with strong swirling flow.

In this work, the authors used the $C F D$ approach to evaluate numerically the thermal exchange, the separation efficiency and the influence of the grain size on the cyclone performance. Six simulations were executed on models differing each other by geometrical characteristics. The simulated case \#1 is the reference case, that is, the model that reproduces the geometry of the system currently in operation. All other cases differ from each other by the vortex finder length.

\subsection{Structure and contribution of the present work}

The complex plant studied in the present paper is an assembly of cyclones as a part of a process whose industrial goal is the production of the cement. The $C F D$ simulations aim to evaluate the particles separation performance and the thermal exchange between the continuous and solid phase are the main physical quantities to improve. This type of problem can be classified as a multiphase system with heat exchange where the air is the continuous phase, at a temperature of $850^{\circ} \mathrm{C}$ and the dispersed phase at $600^{\circ} \mathrm{C}$ consisting, mostly, of calcium carbonate (density equal to $2,063 \mathrm{~kg} / \mathrm{m}^{3}$ ), subsequently intended to the so-called baking 
process, in order to produce clinker and then cement. The volume fraction (VF) of particles, in the simulated domain, ranges from 0 to 0.57 ; as shown in the following paragraphs, the maximum value is reached only in two small regions, where the geometric conformation allows the retention of solid material.

The study was divided into three basic stages: in the first phase, the so-called tuning phase, some simulations were carried out with the aim of obtaining results in agreement with the available experimental data; these simulations were executed on the reference case \#1, characterized by the $v f$ length equal to zero. This case reproduces the geometry of the system currently in operation. During this iterative phase, the quality of the mesh and the model setup were modified to match the main experimental quantities, that is, exit temperature and separation performance.

Using the same setup, consolidated during the previous phase, the work continues through three series of six simulations. Each series changes the $v f$ length of one or more cyclones as summarized in the Tables $1-3$. In very short terms, as detailed in the next sections, increasing the $v f$ length, the solid separation efficiency increases in all cases up to a maximum of $1.5 \%$. At the same time, taking into account the case \#1 as reference case ( $v f$ length $=0$ ), the exit temperature (obviously equal for the two species), presents a modest decrease with a maximum difference equal to $13^{\circ} \mathrm{C}$ and a very modest increase of pressure drop with a max difference equal to 14 Pascal.

Table 1: Separation efficiency varying the $v f$ length of the upper cyclone.

\begin{tabular}{lcc}
\hline & & Separation efficiency \\
& & $\sigma=1-\frac{m_{\text {top exit }}^{\text {solid }}}{m_{\text {total input }}^{\text {solid }}}$ \\
\hline Case & upper $v f$ length $[m]$ & 0.927 \\
$\# 2$ & 0 & 0.930 \\
$\# 3$ & 0.44 & 0.934 \\
$\# 4$ & 0.87 & 0.934 \\
$\# 5$ & 1.31 & 0.936 \\
\hline 6 & 1.73 & 0.931 \\
\hline
\end{tabular}

Table 2: Separation efficiency varying the $v f$ length of the two lower cyclones.

\begin{tabular}{|c|c|c|}
\hline Case & Lower $v f$ length $[m]$ & $\begin{array}{l}\text { Separation efficiency } \\
\qquad \sigma=1-\frac{m_{\text {top exit }}^{\text {sslid }}}{m_{\text {total input }}^{\prime s \text { in }}}\end{array}$ \\
\hline \#1 & 0 & 0.927 \\
\hline$\# 2$ & 0.37 & 0.930 \\
\hline \#3 & 0.75 & 0.930 \\
\hline \#4 & 1.12 & 0.930 \\
\hline \#5 & 1.50 & 0.932 \\
\hline \#6 & 2.25 & 0.941 \\
\hline
\end{tabular}


Table 3: Separation efficiency varying the $v f$ length of all cyclones.

\begin{tabular}{lccc}
\hline & & & Separation efficiency \\
& & & \\
Case & Upper $v f$ length $[m]$ & Lower $v f$ length $[m]$ & $m_{\text {top exit }}^{\text {solid }}$ \\
\hline \#1 & 0 & 0 & 0.927 \\
$m_{\text {total input }}^{\text {solid }}$ & \\
$\# 2$ & 0.44 & 0.37 & 0.934 \\
$\# 4$ & 0.87 & 0.75 & 0.936 \\
$\# 5$ & 1.31 & 1.12 & 0.936 \\
$\# 6$ & 1.73 & 1.50 & 0.937 \\
\hline & 2.60 & 2.25 & 0.937
\end{tabular}

(a)

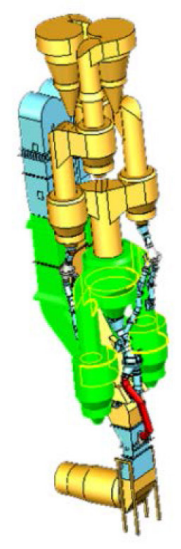

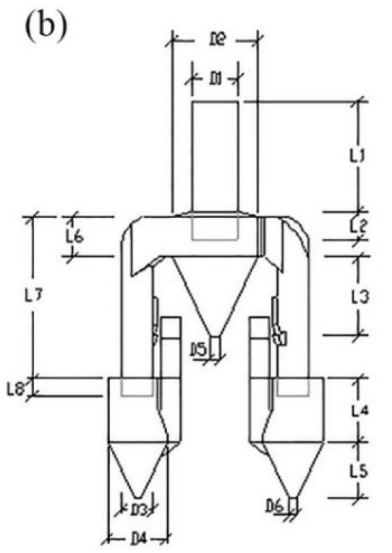

(c)

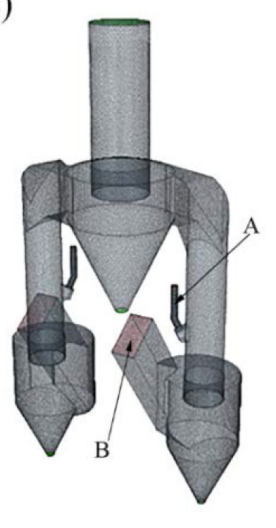

Figure 1: Geometry (a) and mesh representation (b) of the solid separator.

The last part of the work evaluates the effect of the particle grain size on the main performance parameters of the cyclone. To this aim, they have been simulated three cases with particle diameter equal to $30 \mu, 63 \mu$ and $120 \mu$, corresponding to the cases \#5A, \#5 and \#5B, respectively; $63 \mu$ is the mean particle diameter value in the real plant. For these simulations, between the previous six simulations of Table 3, it was chosen the geometry of the case \#5, as a good compromise between the values of the separation efficiency and the exit temperature. The results show that the increase of the particle diameter produces an improvement of the separation efficiency, up to $26 \%$, between the cases \#5A and \#5B, with interesting results also for the exit temperature and for the pressure drop.

\section{MODEL}

\subsection{Cyclone separator}

Together with the Table 4, Fig. 1b shows the geometrical dimensions of the cyclone separator model. Figure 1c shows the volumetric model where A and B represent the main and 
Table 4: Geometry of the cyclone.

\begin{tabular}{lll}
\hline Parameter & Symbol & Size $[\mathrm{mm}]$ \\
\hline Diameter of upper vortex finder & D1 & 3,370 \\
Diameter of upper cylindrical part & D2 & 6,250 \\
Diameter of lower vortex finder & D3 & 2,320 \\
Diameter of lower cylindrical part & D4 & 4,330 \\
Diameter of upper spigot & D5 & 780 \\
Diameter of lower spigot & D6 & 500 \\
Length of upper outlet tube & L1 & 8,014 \\
Length of upper vortex finder & L2 & $*$ \\
Length of upper conical part & L3 & 5,670 \\
Length of lower cylindrical part & L4 & 4,583 \\
Length of lower conical part & L5 & 4,119 \\
Length of upper cylindrical part & L6 & 2,891 \\
Length of lower outlet tube & L7 & 11,700 \\
Length of lower vortex finder & L8 & $*$ \\
\hline
\end{tabular}

*vortex finder length: see Table 5

secondary feed respectively; the same figure shows the mesh constituted by about 500,000 polyhedral volumetric cells with a base size of $10 \mathrm{~cm}$, a minimum cell size of $2 \mathrm{~cm}$ and 2 prism layers at the walls. The transparent visualization enables to see the presence of the vortex finders, the length of which characterizes the six simulated models.

\subsection{Numerical model}

For incompressible, unsteady and non-isothermal flow in cyclone separator, the Eulerian Multiphase model has been used. This model treats a dispersed multiphase flow as two (or more) fully interpenetrating quasi-fluid and also considers the interpenetrating effect of each phase by using drag model. The VF of each phase is defined on the basis of the distribution of each phase and the size of computational volume. The two-fluid model equation for each phase is given by the following equations, where the subscript $k$ represents the single phase:

$$
\begin{gathered}
\sum_{k}^{N} a_{k}=1 \\
\frac{\partial \alpha_{k} \rho_{k}}{\partial t}+\frac{\partial \alpha_{k} \rho_{k} U_{i, k}}{\partial x_{i}}=-\sum_{l=1}^{p}\left(\dot{m}_{k l}-\dot{m}_{l k}\right) \\
\frac{\partial \alpha_{k} \rho_{k} U_{i, k}}{\partial t}+\frac{\partial \alpha_{k} \rho_{k} U_{i, k} U_{j, k}}{\partial x_{j}}=-\alpha_{k} \frac{\partial P}{\partial x_{i}}+\frac{\partial \alpha_{k} \tau_{i j, k}}{\partial x_{k}}+a_{k} \rho_{k} g_{i}+F_{i, k}
\end{gathered}
$$

The difficulty of solve these sets of equations is twofold. First, these equations are difficult from a numerical point of view, since there are many coupled equations with one shared pressure. Secondly, to solve the equations, closure models are required for $\tau$ and $F_{k}$, which represent respectively the rheology of the phase and the interaction force acting on the phase $k$. 
In particular, the drag force $F_{i, k}^{D}$ has been modelling as:

$$
\left.\left.F_{(i, k)}^{D}=A_{(} i, k\right)\left(v_{(} k, i\right)-v_{i}\right)
$$

where the linearized drag coefficient $A_{i k}$ is defined by the Gidaspow model as:

$$
A_{i, k}=\left\{\begin{array}{c}
\frac{150 a_{d}^{2} \mu_{c}}{a_{c} l_{c d}^{2}}+\frac{1,75 a_{d} \rho_{c}\left|v_{r}\right|}{l_{c d}} a_{d} \geq a_{t r} \\
\frac{3}{4} C_{D} \frac{a_{c} \rho_{d}}{l_{c d}}\left|v_{r}\right| a_{c}^{n} a_{d}<a_{t r}
\end{array}\right.
$$

and where the drag coefficient $C_{D}$ is defined by the Schiller-Neumann equation as:

$$
C_{D}=\left\{\begin{array}{c}
\frac{24}{R e_{d}}\left(1+0,15 R e_{d}^{0.687}\right) 0<R e_{d} \leq 1000 \\
0,44 R e_{d}>1000
\end{array}\right.
$$

In this work the transition VF $\alpha_{t r}$ in set to 0.2 and the hindered settling exponent is $n=-1.65$. A 2-Layer Realizable $K-\varepsilon$ model has been applied to the gas phase and a Modified Johnson Frictional model was used to evaluate the stresses in granular medium.

At present, in literature, it is proved that the most utilized turbulence modelling is the RSM turbulence model [1, 3, 8-14]. In addition, all these papers simulate isothermal plants while, the present work has to take into account the thermal exchange in its various forms (convection and radiation). Then, to have an acceptable time of simulation, with the computing power today available, the use of the K- $\varepsilon$ model has been a mandatory choice. For the problem under study, surely, the LES turbulent model is the most suitable one, but at the present, it results a prohibitive approach being a very time consuming model.

Six geometries of the cyclone separator, with six types of outlet configurations, were carried out. The $\mathrm{CCM}+9.6$ package [18] was used to solve the equations by the finite volume method. The boundary conditions concerning the feed of the cyclone (A and B in Fig. 3c) are summarized in Table 5

\section{RESULTS AND DISCUSSIONS}

Tables 1-3and 6 summarize the results computed in 18 different simulated cases. Obviously, the reference case \#1 is repeated in the Table 1-3.

The simulation \#1 (with $v f=0$ ) reproduces exactly the system currently in operation; the experimental values of temperature at the exit, pressure drop and separation efficiency differ from the simulated one no more than $1.5 \%$.

All other simulations of the Tables 1-3, which differ from \#1 only for the $v f$ length, if compared to the case \#1, present an increase in efficiency. Furthermore, it is observed a mod-

Table 5: Boundary conditions (see Fig. 3b).

\begin{tabular}{lccc}
\hline Inlet port & $\begin{array}{c}\text { Gas mass flow rate } \\
{[\mathrm{kg} / \mathrm{s}]}\end{array}$ & $\begin{array}{c}\text { Solid mass flow rate } \\
{[\mathrm{kg} / \mathrm{s}]}\end{array}$ & $\begin{array}{c}\text { Gas/solid temperature. } \\
{\left[{ }^{\circ} \mathrm{C}\right]}\end{array}$ \\
\hline $\mathrm{A}$ & 31 & 25.5 & 850 \\
$\mathrm{~B}$ & 36 & 23.5 & 600 \\
\hline
\end{tabular}


est but monotonous increase of the pressure drop with a maximum difference equal to 14 Pascal and a slight decrease of temperature with a maximum value equal to $13^{\circ} \mathrm{C}$. The greater is the vortex finder length the greater is the number of particles that bumps against its wall. The frictions and collisions are the basis of decrease of particles velocity, then promoting their collection. The slight decrease of temperature at the exit is due to the simultaneous increase of the contact time of the two feeds caused by a greater average particle path, before their expulsion. It is also obvious the reason of the modest increase of the pressure drop that it is the effect due to the increasing intrusiveness of the vortex finder.

Each of the following figures represents the particle VF on two sections concerning the simulation \#1 and the case that presents the greater separation efficiency.

In all the previous figures, it is possible to observe the positive effect of the vortex finder on the separation efficiency mainly due to the barrier effect, while, to the other site, there is a negative effect on the pressure drop whose increase is due to the $v f$ intrusiveness.

Figure 4 shows the trend of the exit temperature of the gas and particles. In general, it is possible to conclude that the introduction of the $v f$ produces a benefit in terms of exit temperature decrease. After a minimum the temperature increases, probably because the $v f$ becomes ever more near the cone with a progressive decrease of separation efficiency. Furthermore, Fig. 4 also shows that, in terms of exit temperature, there aren't benefits coming from the presence of $v f$ in the upper cyclone.

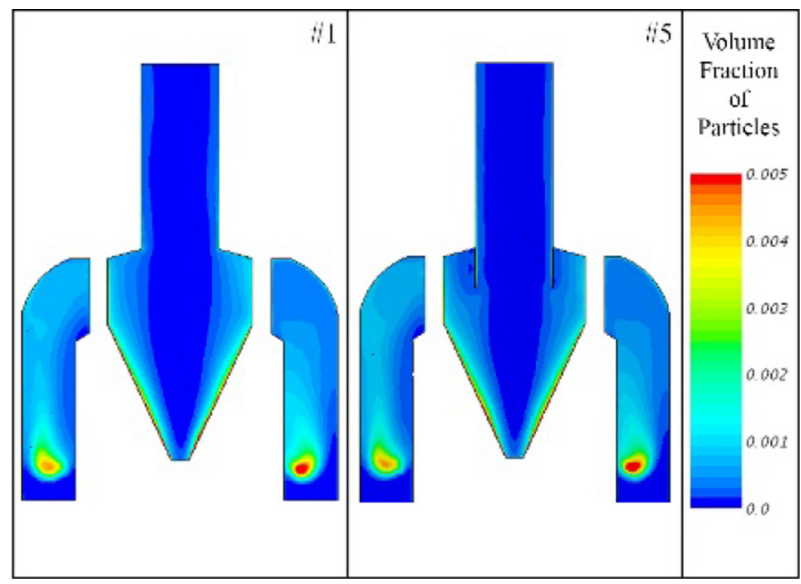

Figure 2: VF 2D-representation concerning the simulations belonging to Table 1 .
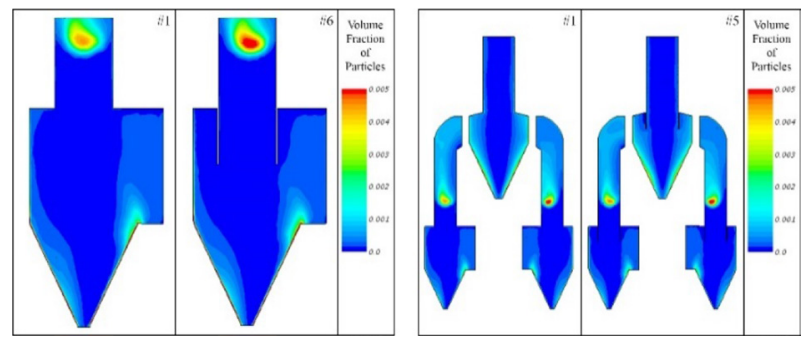

Figure 3: (left) and 4 (right): VF 2D-representations concerning the simulation belonging to Table 2 (left) and 3 (right). 


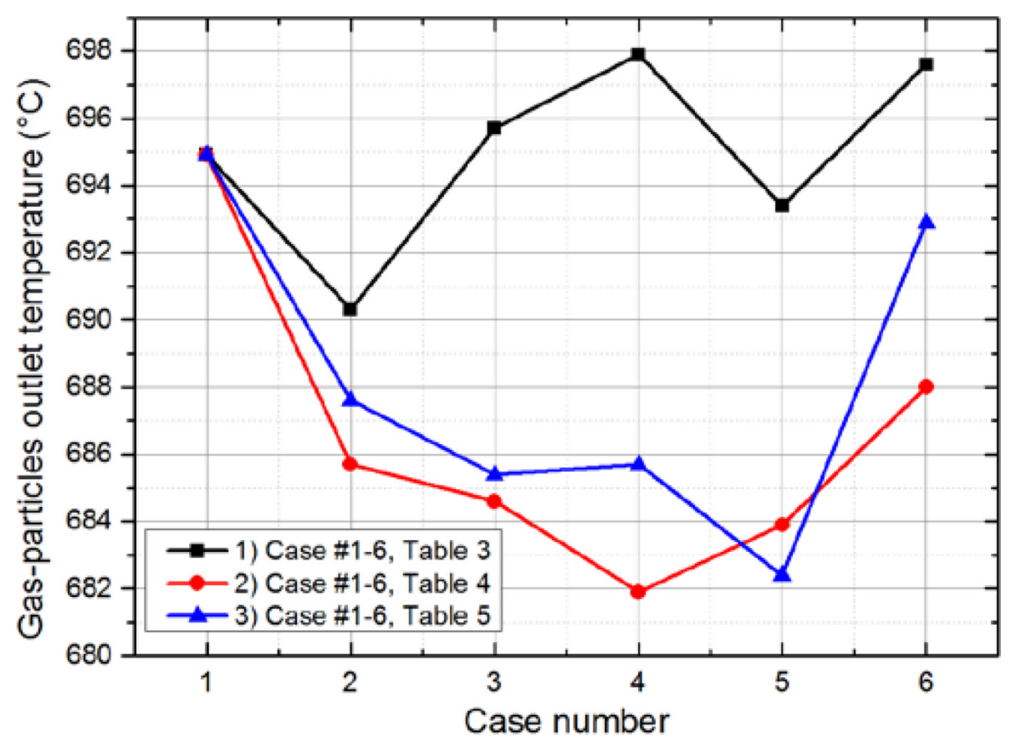

Figure 4: Gas-particle exit temperature trend of the eighteen simulations, grouped by type of geometry.

Table 6: Effect of the particle diameter on the separation efficiency on the temperature at the exit and on the pressure drop.

\begin{tabular}{lcccc}
\hline Case & $\begin{array}{c}\text { Particle } \\
\text { diameter }[\mu](*)\end{array}$ & $\begin{array}{c}\text { Separation } \\
\text { efficiency, }(\sigma)\end{array}$ & $\begin{array}{c}\text { Temperature at the exit } \\
{\left[{ }^{\circ} \mathrm{C}\right]}\end{array}$ & $\begin{array}{c}\text { Pres. drop, } \\
\Delta \mathrm{p},[\mathrm{Pa}]\end{array}$ \\
\hline \#5A & 30 & 0.722 & 722 & 785 \\
$\# 5$ & 63 & 0.937 & 682 & 659 \\
$\# 5 B$ & 120 & 0.984 & 678 & 476 \\
\hline
\end{tabular}

*The simulations \#5A and \#5B use the same geometry of the case \#5 of Table 3; the grain size is the only change.

The simulations \#5A and \#5B implement another parametric study developed by varying the particle grain size. Table 6 compares the two simulations with the previous case \#5. As well known in literature $[1-3,5,6,10,14]$, it highlights that, by increasing the grain size, the separation efficiency increases, while the temperature at the exit and the pressure drop decreases. Gravity, centrifugal forces and friction play a fundamental role on the decrease of kinetic energy of particles and then on the increasing of the efficiency. Assuming a flow constant, the increase of particle size involves a lower number of particles with a consequent increase of gas permeability and then a decrease of pressure drop.

\section{CONCLUSIONS}

The present study is a parametric study that considers, as parameters, the vortex finder length and the particle grain size, alternatively. The experimental values of temperature at the exit, pressure drop and separation efficiency differ, from the simulated case \#1 ( $v f$ length $=0$ ), no more than $1.5 \%$. Case \#1 reproduces the geometry of the system currently in operation. In all 
simulated cases, the greater is the $v f$ length the greater is the solid separation efficiency, while the pressure drop constantly increases of a few Pascals and the exit temperature decreases until a certain value of the $v f$ length and then inverts its trend.

The last part of the work evaluates the effect of the particle grain size on the main performance parameters of the cyclone. To this aim, three cases were simulated with particle diameter equal to $30 \mu, 63 \mu$ and $120 \mu$, corresponding to the cases \#7, \#5 and \#8, respectively. $63 \mu$ is the mean particle diameter value in the operating system. The simulations \#7 and \#8 use the same geometry of the case \#5, chosen as a good compromise between the values of the separation efficiency and the exit temperature,. The increase of the particle diameter results in a better separation efficiency up to $9 \%$, in the case \# 8, with a consequent low variation percent of the exit temperature and a considerable decrease of the pressure drop. It is in progress an activity of simulation, of the same plant, that considers the simultaneous presence of particles with different diameters according to a suitable distribution function.

\section{ACKNOWLEDGEMENTS}

This work was supported by the precious contribute of Colacem S.p.A, - Gubbio (Italy)

\section{REFERENCES}

[1] Lee, J.W., Yang, H.J. \& Lee, D.Y., Effect of the cylinder shape of a long-coned cyclone on the stable flow-field establishment. Powder Technology, 165, pp. 30-38, 2006. http://dx.doi.org/10.1016/j.powtec.2006.03.011

[2] Lim, K.S., Kim, H.S. \& Lee, K.W., Characteristics of the collection efficiency for a cyclone with different vortex finder shapes. Journal of Aerosol Science, 35, pp. 743 754, 2004. http://dx.doi.org/10.1016/j.jaerosci.2003.12.002

[3] Qian, F., Zhang, J. \& Zhang, M., Effects of the prolonged vertical tube on the separation performance of a cyclone. Journal of Hazardous Materials, 136, pp. 822-829, 2006. http://dx.doi.org/10.1016/j.jhazmat.2006.01.028

[4] Chen, J., Xiaofeng, L., Liu, H. \& Yang, C., Effect of the bottom-contracted and edgesloped vent-pipe on the cyclone separator performance. Chemical Engineering Journal, 129, pp. 85-90, 2007. http://dx.doi.org/10.1016/j.cej.2006.11.005

[5] Zhao, B., Shen, H. \& Kang, Y., Development of a symmetrical spiral inlet to improve cyclone separator performance. Powder Technology, 145, pp. 47-50, 2004. http://dx.doi.org/10.1016/j.powtec.2004.06.001

[6] Lim, K.S., Kwon, S.B. \& Lee, K.W., Characteristics of the collection efficiency for a double inlet cyclone with clean air. Jouranal of Aerosol Science, 34, pp. 1085-1095, 2003. http://dx.doi.org/10.1016/S0021-8502(03)00079-X

[7] Zheng, A., Yaxin, S. \& Wan, X., Experimental study of a square-shaped separator with different inlet forms. Journal of Engineering Thermal Energy Power, 23, pp. 293-297, 2008.

[8] Bernardo, S., Mori, M., Peres, A.P. \& Dionisio, R.P., 3-D computational fluid dynamics for gas and gas-particle flows in a cyclone with different inlet section angles. Powder Technology, 162, pp. 190-200, 2006.

http://dx.doi.org/10.1016/j.powtec.2005.11.007 
[9] Wang, B., Xu, D.L., Chu, K.W. \& Yu, A.B., Numerical study of gas-solid flow in a cyclone separator. Applied Mathematical Modelling, 30, pp. 1326-1342, 2006. http://dx.doi.org/10.1016/j.apm.2006.03.011

[10] Fuat, K. \& Irfan, K., Numerical investigation of performance characteristics of a cyclone prolonged with a dipleg. Chemical Engineering Journal, 151, pp. 39-45, 2009. http://dx.doi.org/10.1016/j.cej.2009.01.040

[11] Udaya Bhaskar, K., Rama Murthy, Y., Ravi Raju, M., Tiwari, S., Srivastava, J.K. \& Ramakrishnan, N., CFD simulation and experimental validation studies on hydrocyclone. Minerals Engineering, 20, pp. 60-71, 2007. http://dx.doi.org/10.1016/j.mineng.2006.04.012

[12] Xiang, R.B. \& Lee, K.W., Numerical study of flow field in cyclones of different height. Chemical Engineering and Processing: Process Intensification, 44, pp. 877-883, 2005. http://dx.doi.org/10.1016/j.cep.2004.09.006

[13] Hoekstra, A.J., Derksen, J.J. \& Van Den Akker, H.E.A., An experimental and umerical study of turbulent swirling flow in gas cyclones. Chemical Engineering Science, 54, pp. 2055-2056, 1999. http://dx.doi.org/10.1016/S0009-2509(98)00373-X

[14] Pant, K., Crowe, C.T. \& Irving, P., On the design of miniature cyclones for the collection of bioaerosols. Powder Technol, 125(2-3), pp. 260-265, 2002. http://dx.doi.org/10.1016/S0032-5910(01)00514-9

[15] Sommerfeld, M., Ho, C.H., Numerical calculation of particle transport in turbulent wall bounded flows. Powder Technology, 131, pp, 1-6, 2003.

[16] Junfu, L., Jiansheng, Z., Hai, Z., Qing, L. \& Guagxi, Y., Performance evaluation of a 220 t/h CFB boiler with water-cooled square cyclones. Fuel Processing Technology, 88, pp. 129-135, 2007. http://dx.doi.org/10.1016/j.fuproc.2004.12.008

[17] Su, Y., Zheng, A. \& Zhao, B., Numerical simulation of effect of inlet configuration on square cyclone separator performance, Powder Technology, 210, pp. 293-303, 2011. http://dx.doi.org/10.1016/j.powtec.2011.03.034

[18] CCM+ User's Guide 9.06, CD-Adapco. 